\title{
GAUSS-CHEBYSHEV QUADRATURE FORMULAE FOR STRONGLY SINGULAR INTEGRALS
}

BY

\author{
ALEXANDER M. KORSUNSKY
}

Department of Materials Science and Metallurgy, University of Cambridge, England

\begin{abstract}
This paper presents some explicit results concerning an extension of the mechanical quadrature technique, namely, the Gauss-Jacobi numerical integration scheme, to the class of integrals whose kernels exhibit second order of singularity (i.e., one degree more singular than Cauchy). In order to ascribe numerical values to these integrals they must be understood in Hadamard's finite-part sense. The quadrature formulae are derived from those for Cauchy singular integrals.

The resulting discretizations are valid at a number of fixed points, determined as the zeroes of a certain Jacobi polynomial. As in all Gaussian quadratures, the final quadrature formulae involve fixed nodal points and provide exact results for polynomials of degree $2 n-1$, where $n$ is the number of nodes. These properties make this approach rather attractive for applications to fracture mechanics problems, where often numerical solution of integral equations with strongly singular kernels is the objective. Numerical examples of the application of the Gauss-Chebyshev rule to some plane and axisymmetric crack problems are given.
\end{abstract}

1. Introduction. The problem of numerical quadrature for the integrals with kernels possessing a singularity of the order exceeding one, i.e., stronger than Cauchy, is quite common in potential theory and the associated problems in continuum mechanics. An example is when a crack in two-dimensional elasticity is modelled using a distribution of dislocation dipoles. The stress $\sigma(x)$ arising at a point $x$ due to a dipole at point $t$ may be expressed as

$$
\mathbf{K}(t, x)=\frac{D}{(t-x)^{2}}+k(t, x)
$$

where $D$ is a constant depending on the elastic parameters and the dipole intensity. Depending on the exact nature of the problem and that of the domain, $k(t, x)$ may contain Cauchy (first-order) or logarithmic singularities, as well as regular terms. For a

Received August 4, 1995.

1991 Mathematics Subject Classification. Primary 65D32.

Current address: Department of Mechanical, Materials, and Manufacturing Engineering, S4B Stephenson Building, University of Newcastle, Newcastle upon Tyne, NE1 7RU, England. 
given distribution $g(t)$ of dipoles over a finite interval $(a, b)$ the overall stress is formally given by

$$
\sigma(x)=f_{a}^{b} K(t ; x) g(t) d t=\int_{a}^{b} \frac{D g(t)}{(t-x)^{2}} d t+\int_{a}^{b} g(t) k(t, x) d t .
$$

While the second integral with the kernel $k(t, x)$ converges at least in the Cauchy principal value sense, the first integral above must be interpreted in the Hadamard finite part sense [6]. The question of developing a consistent interpretation of highly singular integrals based on the theory of generalized functions has been addressed in some detail $[16,5]$.

An excellent overview of numerical quadratures for hypersingular integrals is given in the paper by Monegato [17]. It provides an up-to-date list of various numerical integration schemes developed for this class of problems. The paper dwells in some detail on convergence estimates for various quadrature rules and discusses their relative merits from this aspect. However, another important property distinguishing some techniques from the others is their suitability for the numerical solution of integral equations.

Kutt $[14,15]$ has developed quadrature formulae for the integrals

$$
\int_{a}^{b} \frac{g(t) d t}{(t-x)^{\lambda}}
$$

where $\lambda$ may be greater than unity. However, these involve equally spaced stations depending on the choice of $x$. This turns out to be a strong limitation for potential application to the solution of boundary integral equations.

An approach introduced by Kaya and Erdogan [10] relies on the extraction of the strongly singular part of the kernel, which is then integrated analytically. The remainder of the kernel is treated numerically. This approach leads to very good results, with the only caveat that when the overall kernel cannot be explicitly given in terms of a sum of the hypersingular part and a remainder, the extraction of a strong singularity may lead to a loss of accuracy.

Paget [20] derived a formula for the finite-part integral (2) that involves at least first and possibly further derivatives of the function $g(t)$. This circumstance may be viewed as an obstacle when the unknown function $g(t)$ needs to be determined. Similar comment may be made about the quadrature given by Ioakimidis and Theocaris [8].

As noted by Monegato [17], free from this drawback are formulae of interpolatory type, i.e., quadratures obtained by replacing $g(t)$ by its Lagrange interpolation polynomial over a set of distinct nodes. Interpolatory quadrature formulae

$$
f_{-1}^{1} \frac{w(t) g(t) d t}{t-x} \simeq \sum_{i=1}^{n} \mathrm{w}_{n i}(x) g\left(t_{n i}\right)
$$

have been analysed in much detail in [18] for Cauchy integrals. Also considered were formulae for their derivatives with respect to $x$, i.e., finite-part integrals

$$
\frac{d}{d x} f_{-1}^{1} \frac{w(t) g(t) d t}{t-x}=f_{-1}^{1} \frac{w(t) g(t) d t}{(t-x)^{2}} \simeq \sum_{i=1}^{n} \mathrm{w}_{n i}^{\prime}(x) g\left(t_{n i}\right) .
$$

General expressions were given for integrals involving weight functions corresponding to various Jacobi polynomials. A comparison between the general result in [18] and the 
special case considered in this paper is made and agreement between the formulae is found $(\S 4)$.

In this paper an explicit procedure is presented that demonstrates the derivation of the Gauss-Chebyshev rule for finite-part integrals from the corresponding Cauchy integral quadrature formulae. The resulting discretized form of the integral is given by a sum over an arbitrary number $n$ of distinct nodes, determined as the roots of the Chebyshev polynomial $U_{n}(t)$. The corresponding weight function $w(t)=\sqrt{1-t^{2}}$ is encountered in numerous fracture mechanics problems and describes the crack opening displacement in the vicinity of the crack front. It is important that the resulting formula may be used uniformly for explicit or implicit kernels, as well as for those containing a Cauchy singularity alongside the second-order term. This approach naturally lends itself to an effective and simple procedure for the solution of the associated integral equations arising in many fracture mechanics problems.

2. Principal value and finite-part integrals. In the development of the quadrature formulae the following relations form the basis [22]:

$$
\begin{array}{r}
\frac{1}{\pi} Q_{n}^{(\alpha, \beta)}(x)=\frac{1}{\pi} \int_{-1}^{1} P_{n}^{(\alpha, \beta)}(t) w(t) \frac{d t}{t-x}=-\frac{\Gamma(\alpha) \Gamma(1-\alpha)}{\pi} 2^{-k} P_{n-k}^{(-\alpha,-\beta)}(x) \\
(-1<x<1)
\end{array}
$$

where $w(t)$ is the weight function appropriate for the Jacobi polynomials $P_{n}^{(\alpha, \beta)}(t)$,

$$
w(t)=(1-t)^{\alpha}(1+t)^{\beta} \quad(-1<\alpha, \beta<1,-1<t<1)
$$

and $k=-(\alpha+\beta)$ is an integer that may assume values $-1,0$, and 1 .

In particular, if $\alpha=\beta=1 / 2$, the Jacobi polynomials under the integral sign are related to the Chebyshev polynomials of the second kind $U_{n}(t)$ by

$$
P_{n}^{\left(\frac{1}{2}, \frac{1}{2}\right)}(t)=\frac{2 \Gamma\left(n+\frac{3}{2}\right)}{(n+1) ! \sqrt{\pi}} U_{n}(t),
$$

and the Jacobi polynomials on the right-hand side of Eq. (6) are related to the Chebyshev polynomials of the first kind $T_{n}(x)$ by

$$
P_{n}^{\left(-\frac{1}{2},-\frac{1}{2}\right)}(t)=\frac{\Gamma\left(n+\frac{1}{2}\right)}{n ! \sqrt{\pi}} T_{n}(x) .
$$

For this particular choice of $\alpha, \beta$, Eq. (6) may be recast using Chebyshev polynomials in the form

$$
f_{-1}^{1} \frac{U_{n}(t) \sqrt{1-t^{2}} d t}{(t-x)}=-T_{n+1}(x) \quad(-1<x<1) .
$$

The following differentiation formula will be found useful:

$$
\frac{d^{m}}{d x^{m}} P_{n}^{(\alpha, \beta)}(x)=\frac{1}{2^{m}} \frac{\Gamma(n+m+\alpha+\beta+1)}{\Gamma(n+\alpha+\beta+1)} P_{n-m}^{(\alpha+m, \beta+m)}(x),
$$


which for Chebyshev polynomials of the first and second kind becomes [22]

$$
\begin{aligned}
T_{n+1}^{\prime}(x) & =(n+1) U_{n}(x), \\
U_{n}^{\prime}(x) & =\left(1-x^{2}\right)^{-1}\left[(n+1) T_{n+1}(x)+x U_{n}(x)\right] .
\end{aligned}
$$

Now, going back to Eq. (6) we note the following. Let us formally differentiate both sides of this relationship with respect to $x$, and change the order of differentiation and integration on the left-hand side. The resulting expression on the right-hand side then becomes the value of the integral on the left, understood in the finite-part sense. Therefore

$$
\frac{1}{\pi} f_{-1}^{1} P_{n}^{(\alpha, \beta)}(t) w(t) \frac{d t}{(t-x)^{2}}=-\frac{\Gamma(\alpha) \Gamma(1-\alpha)}{\pi} 2^{-k} \frac{d}{d x} P_{n-k}^{(-\alpha,-\beta)}(x) \quad(-1<x<1) .
$$

In particular, for the Chebyshev polynomial case $\left(\alpha=\beta=\frac{1}{2}\right)$, using the differentiation formulae for $T_{n}(x)(12)$, one obtains

$$
f_{-1}^{1} \frac{U_{n}(t) \sqrt{1-t^{2}} d t}{(t-x)^{2}}=-(n+1) U_{n}(x) \quad(-1<x<1) .
$$

3. Cauchy kernels. The initial stage of the quadrature development follows the pattern presented in [4] for principal value (Cauchy) integrals. For this reason, in this section, the part of reasoning that is common to the development of both Cauchy singular and second-order singular integrals is given for completeness, with some additional clarifications given where appropriate. The next section then demonstrates how this technique may be modified and adapted to hypersingular integrals specifically.

Consider the following integral:

$$
S(x)=\frac{1}{\pi} f_{-1}^{1} g(t) w(t) \frac{d t}{t-x} \quad(-1<x<1)
$$

where $g(t)$ is bounded for $-1 \leq t \leq 1$, and $w(t)$ is given by (7). Let us assume next that the function $g(t)$ may be approximated with a sufficient degree of accuracy by a truncated series of Jacobi polynomials, corresponding to the weight function $w(t)$,

$$
g(t) \simeq \sum_{j=0}^{p} B_{j} P_{j}^{(\alpha, \beta)}(t) \quad(-1<t<1) .
$$

From Eq. (16) it then follows that

$$
S(x)=C \sum_{j=0}^{p} B_{j} P_{j}^{(-\alpha .-\beta)}(x),
$$

where $C$ is a constant independent of $j$, which is determined from (6).

As the next step, a fraction expansion of the Jacobi polynomials $P_{j}^{(-\alpha,-\beta)}(x)$, appearing on the right-hand side, in terms of the polynomials $P_{j}^{(\alpha, \beta)}(t)$ on the left-hand side, is constructed. The general formula sought at this point has the following form:

$$
-\frac{P_{j}^{(\alpha, \beta)}(x) P_{n}^{(-\alpha,-\beta)}(x) \pm P_{j}^{(-\alpha,-\beta)}(x) P_{n}^{(\alpha, \beta)}(x)}{P_{n}^{(\alpha, \beta)}(x)}=\sum_{i=1}^{n} \frac{a_{i}}{t_{i}-x}, \quad j<n .
$$


Here the sum is taken over the zeroes of the Jacobi polynomial $P_{n}^{(\alpha, \beta)}(x)$. With the appropriate choice of stations and collocation points this expansion may then be interpreted as a mechanical quadrature.

It is natural to specialize the further development of the method to particular values of $\alpha$ and $\beta$, since the fraction expansion depends on the nature of the set of Jacobi polynomials used. Assume $\alpha=\beta=\frac{1}{2}$, and search for the coefficients $a_{i}$ in the following identity:

$$
-\frac{T_{j+1}(x) U_{n}(x)-T_{n+1}(x) U_{j}(x)}{U_{n}(x)}=\sum_{i=1}^{n} \frac{a_{i}}{t_{i}-x}, \quad j<n, U_{n}\left(t_{i}\right)=0 .
$$

To verify that the partial fraction expansion above is possible, the left-hand side may be expressed in an alternative way using the recursion formulae [3]:

$$
U_{q-p-1}(x)=T_{p}(x) U_{q-1}(x)-T_{q}(x) U_{p-1}(x) .
$$

Then, taking $p=i, q=k$ and substituting into Eq. (20) it is found that

$$
\sum_{i=1}^{n} \frac{a_{i}}{t_{i}-x}=-\frac{U_{n-j-1}(x)}{U_{n}(x)}
$$

so that the degree of the numerator is less than that of the denominator, and expansion is possible.

In order to find the values of $a_{i}$ it is convenient to multiply both sides of Eq. (20) by

$$
\left(x-t_{1}\right)\left(x-t_{2}\right) \cdots\left(x-t_{i-1}\right)\left(x-t_{i}\right)\left(x-t_{i+1}\right) \cdots\left(x-t_{n}\right)=U_{n}(x)
$$

and substitute $x=t_{i}$. Then, noting

$$
\left(t_{i}-t_{1}\right) \cdots\left(t_{i}-t_{i-1}\right)\left(t_{i}-t_{i+1}\right) \cdots\left(t_{i}-t_{n}\right)=U_{n}^{\prime}\left(t_{i}\right)
$$

one obtains

$$
a_{i}=-\frac{T_{n+1}\left(t_{i}\right) U_{j}\left(t_{i}\right)}{U_{n}^{\prime}\left(t_{i}\right)} .
$$

Using the differentiation rule (13) for points $t_{i}$ such that $U_{n}\left(t_{i}\right)=0$, this simplifies to

$$
a_{i}=\frac{\left(1-t_{i}^{2}\right)}{(n+1)} U_{j}\left(t_{i}\right)
$$

so that the resulting form of the fraction expansion (20) is

$$
-\frac{T_{j+1}(x) U_{n}(x)-T_{n+1}(x) U_{j}(x)}{U_{n}(x)}=\sum_{i=1}^{n} \frac{1-t_{i}^{2}}{(n+1)\left(t_{i}-x\right)} U_{j}\left(t_{i}\right) .
$$

Now let us select a discrete set of points $x_{k}, k=1, \ldots, n+1$ such that

$$
T_{n+1}(x)=0 ;
$$

then

$$
\sum_{i=1}^{n} \frac{\left(1-t_{i}^{2}\right)}{(n+1)} \frac{U_{j}\left(t_{i}\right)}{t_{i}-x_{k}}=-T_{j+1}\left(x_{k}\right)
$$


Now, multiplying both sides by $B_{j}$, noting that for Chebyshev polynomials in Eq. (18) $C=-1$, and changing the order of summation,

$$
S\left(x_{k}\right)=-\sum_{j=0}^{p} B_{j} T_{j+1}\left(x_{k}\right)=\sum_{i=1}^{n} \frac{\left(1-t_{i}^{2}\right)}{(n+1)} \frac{g\left(t_{i}\right)}{t_{i}-x_{k}} .
$$

The following mechanical quadrature ensues:

$$
\frac{1}{\pi} \int_{-1}^{1} \frac{\sqrt{1-t_{i}^{2}} g\left(t_{i}\right) d t}{t_{i}-x_{k}} \simeq \sum_{i=1}^{n} \frac{\left(1-t_{i}^{2}\right)}{(n+1)} \frac{g\left(t_{i}\right)}{t_{i}-x_{k}} .
$$

It is valid only at a number of fixed discrete points $x_{k}: T_{n+1}\left(x_{k}\right)=0$.

The Gauss-Chebyshev integration formula for a bounded continuous function $f(t, x)$, where $x$ is a parameter, is given by

$$
\frac{1}{\pi} \int_{-1}^{1} f\left(t, x_{0}\right) \sqrt{1-t^{2}} d t \simeq \sum_{i=1}^{n} \frac{\left(1-t_{i}^{2}\right)}{(n+1)} f\left(t_{i}, x_{0}\right) .
$$

Note that this formula may be applied to determine the values of the integral on the left-hand side for an arbitrary value of the parameter $x=x_{0}$. Also, the weights are identical with those appearing in Eq. (30). Therefore, a more general type of integral over $t$ of a function $f(t, x)$ may be considered, and a mechanical quadrature formula may be obtained by combining the above results giving

$$
S\left(x_{k}\right)=\frac{1}{\pi} \int_{-1}^{1} f(t, x) \sqrt{1-t^{2}} d t \simeq \sum_{i=1}^{n} \frac{\left(1-t_{i}^{2}\right)}{(n+1)} f\left(t_{i}, x_{k}\right) .
$$

Here, the values of $x_{k}$ must be chosen according to (27), and the function $f(t, x)$ may contain first-order singularities as $(t-x) \rightarrow 0$, i.e., one may write

$$
f(t, x)=\frac{g(t)}{t-x}+k(t, x)
$$

where $k(t, x)$ is a regular function.

4. Second-order singular kernels. It is now our intention to extend these results to the next order of singularity, i.e., to derive a mechanical quadrature formula for the integral

$$
S(x)=\frac{1}{\pi} f_{-1}^{1} g(t) w(t) \frac{d t}{(t-x)^{2}} \quad(-1<x<1) .
$$

We proceed in a fashion entirely similar to that of the previous section, i.e., assume

$$
g(t) \simeq \sum_{j=0}^{p} B_{j} U_{j}(t), \quad S(x) \simeq-\sum_{j=0}^{p} B_{j} T_{j+1}^{\prime}(x) .
$$

We then proceed directly to Eq. (26), which holds for an arbitrary $x$ in $(-1,1)$ and may be differentiated once with respect to $x$ to give:

$$
\sum_{i=1}^{n} \frac{\left(1-t_{i}^{2}\right)}{(n+1)} \frac{U_{j}\left(t_{i}\right)}{\left(t_{i}-x\right)^{2}}=-T_{j+1}^{\prime}(x)+\frac{d}{d x}\left[\frac{T_{n+1}(x) U_{j}(x)}{U_{n}(x)}\right] .
$$


In order to preserve the consistency of the final formula with that for Cauchy singular kernels, the collocation points $x$ must once again be chosen as zeroes of the Chebyshev polynomial $T_{n+1}(x)$, yielding

$$
\sum_{i=1}^{n} \frac{\left(1-t_{i}^{2}\right)}{(n+1)} \frac{U_{j}\left(t_{i}\right)}{\left(t_{i}-x_{k}\right)^{2}}=-T_{j+1}^{\prime}\left(x_{k}\right)+\frac{U_{j}\left(x_{k}\right)}{U_{n}\left(x_{k}\right)} T_{n+1}^{\prime}(x),
$$

since the two other terms in the derivative turn to zero. Using the differentiation formula (12) at $x_{k}: T_{n+1}\left(x_{k}\right)=0$ the above relation may be rewritten as

$$
-T_{j+1}^{\prime}\left(x_{k}\right)=\sum_{i=1}^{n} \frac{\left(1-t_{i}^{2}\right)}{(n+1)} \frac{U_{j}\left(t_{i}\right)}{\left(t_{i}-x_{k}\right)^{2}}-(n+1) U_{j}\left(x_{k}\right) .
$$

Both sides of this equation may now be multiplied by the coefficients $B_{j}$ and summed over $j$ from 0 to $p$ to recover

$$
S\left(x_{k}\right) \simeq \sum_{i=1}^{n} \frac{\left(1-t_{i}^{2}\right)}{(n+1)} \frac{g\left(t_{i}\right)}{\left(t_{i}-x_{k}\right)^{2}}-(n+1) g\left(x_{k}\right) .
$$

This expression has the appearance of a discretized record of a Fredholm integral equation of the second kind. If an application of the method to the solution of integral equations is in mind, good convergence to the accurate solution may be expected.

One further step is required, which would lead to the right-hand side being evaluated only at $n$ points $t_{i}: U_{n}\left(t_{i}\right)=0$. It may be achieved with the aid of the Lagrange interpolation formula, which will be exact within the class of polynomials chosen to represent $g(t)[22]$ :

$$
g\left(x_{k}\right)=\sum_{i=1}^{n} g\left(t_{i}\right) \frac{U_{n}\left(x_{k}\right)}{U_{n}^{\prime}\left(t_{i}\right)\left(x_{k}-t_{i}\right)}
$$

which leads to

$$
S\left(x_{k}\right) \simeq \sum_{i=1}^{n} \frac{\left(1-t_{i}^{2}\right)}{(n+1)} \frac{g\left(t_{i}\right)}{\left(t_{i}-x_{k}\right)^{2}}+\sum_{i=1}^{n} \frac{(n+1)}{t_{i}-x_{k}} \frac{U_{n}\left(x_{k}\right)}{U_{n}^{\prime}\left(t_{i}\right)} g\left(t_{i}\right) .
$$

It is interesting to note at this point that the above result is in agreement with the general expression for Gauss-Jacobi weights for second-order integrals given in [18]. Indeed, substituting $\alpha=\beta=1 / 2$ into Eq. (3.2) in [18]

$$
\mathrm{w}_{n i}^{\prime}(x)=\frac{1}{t_{n i}-x}\left[\mathrm{w}_{n i}(x)-\frac{Q_{n}^{(\alpha, \beta) \prime}(x)}{P_{n}^{(\alpha, \beta) \prime}\left(t_{n i}\right)}\right],
$$

it is found that

$$
\mathrm{w}_{n i}^{\prime}(x)=\frac{1}{t_{n i}-x}\left[\mathrm{w}_{n i}(x)+\frac{(n+1) U_{n}(x)}{U_{n}^{\prime}\left(t_{n i}\right)}\right],
$$

i.e., precisely the present result.

Further progress may be achieved, however, if specific properties of the current problem are taken into consideration. Once again using Eq. (13), one finally obtains

$$
S\left(x_{k}\right) \simeq \sum_{i=1}^{n}\left\{\frac{\left(1-t_{i}^{2}\right)}{(n+1)} \frac{1}{\left(t_{i}-x_{k}\right)^{2}}-\frac{\left(1-t_{i}^{2}\right)}{t_{i}-x_{k}} \frac{U_{n}\left(x_{k}\right)}{T_{n+1}\left(t_{i}\right)}\right\} g\left(t_{i}\right) .
$$


This may be simplified further, if specific values are chosen for the points $x_{k}$ and $t_{i}$ :

$$
\begin{gathered}
x_{k}: T_{n+1}\left(x_{k}\right)=0, \quad x_{k}=\cos \left(\frac{(2 k-1) \pi}{2(n+1)}\right), k=1, \ldots, n+1 ; \quad U_{n}\left(x_{k}\right)=\frac{(-1)^{k+1}}{\sqrt{1-x_{k}^{2}}}, \\
t_{i}: U_{n}\left(t_{i}\right)=0, \quad t_{i}=\cos \left(\frac{i \pi}{(n+1)}\right), i=1, \ldots, n ; \quad T_{n+1}\left(t_{i}\right)=(-1)^{i} .
\end{gathered}
$$

The resulting formula has the form

$$
\frac{1}{\pi} \int_{-1}^{1} \frac{\sqrt{1-t^{2}} g(t) d t}{(t-x)^{2}} \simeq \sum_{i=1}^{n}\left\{\frac{\left(1-t_{i}^{2}\right)}{(n+1)} \frac{1}{\left(t_{i}-x_{k}\right)^{2}}+\frac{\left(1-t_{i}^{2}\right)}{t_{i}-x_{k}} \frac{(-1)^{i+k}}{\sqrt{1-x_{k}^{2}}}\right\} g\left(t_{i}\right) .
$$

It is worth noting that in a more general case when the integral kernel may be split into a strongly singular part and the remainder,

$$
K(t, x)=\frac{1}{(t-x)^{2}}+k(t, x)
$$

the formula holds in precisely the same form, i.e.,

$$
\frac{1}{\pi} \int_{-1}^{1} \sqrt{1-t^{2}} g(t) K(t, x) d t \simeq \sum_{i=1}^{n}\left\{\frac{\left(1-t_{i}^{2}\right)}{(n+1)} K\left(t_{i}, x_{k}\right)+\frac{\left(1-t_{i}^{2}\right)}{t_{i}-x_{k}} \frac{(-1)^{i+k}}{\sqrt{1-x_{k}^{2}}}\right\} g\left(t_{i}\right) .
$$

5. Examples. Examples include a straight crack in an infinite elastic plane, a buried crack normal to the surface of an elastic half-plane, and an annular crack under axial tension.

In all cases the equation to be solved assumes the following form:

$$
-\frac{2(1-\nu)}{\mu} \sigma(x)=\frac{1}{\pi} f_{-1}^{1} \sqrt{1-t^{2}} g(t) K(t, x) d t,
$$

where $\mu$ is the shear modulus, $\nu$ is Poisson's ratio, and $\sigma(x)$ is the applied tension at infinity.

The examples have been chosen so that the corresponding kernels $K(t, x)$ exhibit increasing degrees of complexity: a simple second-order kernel, corresponding to a straight crack in an infinite elastic plane; a kernel consisting of the strongly singular and a regular part, for a buried crack normal to the surface of an elastic half-plane; and an implicit kernel, given in terms of a Lipschitz-Hankel integral potential, which may be expanded into a sum of a strongly singular term, a Cauchy term, a logarithmic term and a regular part, for an annular crack subjected to axial tension.

5.1. Crack in an infinite plane. In this case the integral equation is given explicitly by (34), with $S(x)=-(2(1-\nu) / \mu) \sigma(x)$, where $\sigma(x)=\sigma_{0}$ if the crack is subjected to uniform remote tension (or constant internal pressure), or uniform remote shear.

The discretized version of the integral equation is given by (47). This furnishes a system of $n+1$ algebraic equations for the $n$ unknown values $g\left(t_{i}\right)$. Provided there is no rapid stress variation over the middle part of the crack (which is certainly not the case in the problem considered), one superfluous equation corresponding to $x_{k}=0$ may be 
conveniently dropped if $n$ is chosen to be even. The stress intensity factors at crack tips are then determined from the values $g\left(t_{1}\right)$ and $g\left(t_{n}\right)$ using the formulae

$$
\begin{gathered}
K_{i}(a)=\frac{\mu}{2(1-\nu)} \sigma_{0} \sqrt{\pi a} g\left(x_{1}\right), \\
K_{i}(-a)=\frac{\mu}{2(1-\nu)} \sigma_{0} \sqrt{\pi a} g\left(x_{n}\right),
\end{gathered}
$$

where $i=1,2$ depending on the crack opening mode.

5.2. Buried normal crack in a half-plane. In this case (see, for example, [12])

$$
K(t, x)=\frac{1}{(t-x)^{2}}-\frac{1}{(t+x+2 d)^{2}}+\frac{12(t+d)(x+d)}{(t+x+2 d)^{4}}
$$

where the unit of length is half of the crack extension and $d>1$ is the depth of the crack centre in these units, i.e., $K(t, x)$ contains regular terms. The discretized form of the integral is given by (48), and the solution proceeds in a manner that is entirely similar to that of the problem in the previous section. Numerical results for this problem, obtained using an IBM PC-486 and FORTRAN coding, are shown in Table 1 (see p. 470). The overall time required for the solutions was approximately 1.3 seconds for 100 stations and 7 seconds for 200 stations.

5.3. Annular crack under axial tension. Let the crack lie in the plane $z=0$ and occupy the region $\left(r_{c}-1, r_{c}+1\right)$. Let it be represented by an unknown distribution of ring dipoles opening in the $z$-direction. Axial stress at points lying on the plane $z=0$ at radius $r$ due to a ring dipole of unit intensity of radius $a$ is given by [7]

$$
\sigma_{z z}(r, 0)=-\frac{\mu}{2(1-\nu) a^{2}} J(0,0 ; 2)(r / a, 0) .
$$

Here

$$
J(0,0 ; 2)(r / a, z / a)=\int_{0}^{\infty} J_{0}(t) J_{0}(r t / a) t^{2} e^{-z t / a} d t
$$

is a Lipschitz-Hankel integral, where $J_{0}$ is the Bessel function of the first kind. This may be expressed in terms of complete elliptic integrals [2]. In particular, for $z=0$,

$$
J(0,0 ; 2)(r / a, 0)=-\frac{k^{3}}{4 \pi k^{\prime 2}(r / a)^{\frac{3}{2}}} \mathbf{E}(k),
$$

where the elliptic parameter $k=2 \sqrt{r / a} /(1+r / a), k^{\prime}=\sqrt{1-k^{2}}$, and $\mathbf{E}(k)$ is the complete elliptic integral of the second kind. Asymptotic properties of the Lipschitz-Hankel integrals were analysed in much detail $[2,21,11]$. Using these results an asymptotic expansion for $\sigma(x)$ for $t \rightarrow x$ is given by

$$
\sigma(x) \simeq \frac{\mu}{2 \pi(1-\nu)}\left[\frac{1}{(r-a)^{2}}+\frac{1}{2 a(r-a)}+\cdots\right] .
$$

Thus, the kernel $K(t, x)$ has the form required for the application of our quadrature formula, and the discretized version (48) may be applied once again.

The quadrature method was tested for accuracy and speed using an IBM PC-486 (66 $\mathrm{MHz}$ ) and FORTRAN coding. The results were compared to those given by Clemens and Ang [1] and showed excellent agreement. The numerical results for different crack mid-radii are given in Table 2, alongside the values taken from Murakami [19]. Following 
TABLE 1. Stress intensity factors $K_{I} /\left(\sigma_{0} \pi^{1 / 2}\right)$ for a buried crack normal to the surface of a half-plane under uniform remote tension $\sigma_{0}$

\begin{tabular}{|l|l|r|r|}
\hline Crack mid-depth & No. of stations & Upper tip & Lower tip \\
\hline 1.01 & 200 & 3.641 & 1.330 \\
1.05 & 200 & 2.155 & 1.254 \\
1.1 & 200 & 1.759 & 1.211 \\
1.2 & 100 & 1.464 & 1.163 \\
1.3 & 100 & 1.332 & 1.133 \\
1.4 & 100 & 1.254 & 1.112 \\
1.5 & 50 & 1.203 & 1.097 \\
2 & 50 & 1.091 & 1.054 \\
3 & 50 & 1.035 & 1.025 \\
4 & 50 & 1.018 & 1.014 \\
5 & 50 & 1.011 & 1.009 \\
10 & 10 & 1.00264 & 1.00239 \\
20 & 10 & 1.00064 & 1.00061 \\
50 & 10 & 1.00010 & 1.00010 \\
100 & 10 & 1.00003 & 1.00002 \\
\hline
\end{tabular}

TABLE 2. Stress intensity factors $K_{I} /\left(\sigma_{0}\left(\pi\left(r_{c}+1\right)\right)^{1 / 2}\right)$ for an annular crack of mid-radius $r_{c}$ and half-width unity under uniform remote tension $\sigma_{0}$

\begin{tabular}{|l|l|r|r|}
\hline Crack mid-radius $r_{c}$ & No. of stations & Inner tip (Murakami) & Outer tip (Murakami) \\
\hline 1.020202 & 200 & $4.041(4.090)$ & $0.634(0.634)$ \\
1.222222 & 200 & $1.322(1.323)$ & $0.610(0.610)$ \\
1.5 & 200 & $0.950(0.950)$ & $0.580(0.581)$ \\
1.85714 & 100 & $0.775(0.775)$ & $0.548(0.548)$ \\
2.333333 & 100 & $0.659(0.659)$ & $0.513(0.513)$ \\
3 & 100 & $0.568(0.568)$ & $0.473(0.473)$ \\
4 & 100 & $0.488(0.488)$ & $0.427(0.427)$ \\
5.666666 & 100 & $0.410(0.410)$ & $0.374(0.374)$ \\
9 & 100 & $0.327(0.327)$ & $0.309(0.309)$ \\
19 & 100 & $0.227(0.227)$ & $0.221(0.221)$ \\
199 & 100 & $0.071(0.071)$ & $0.071(0.071)$ \\
\hline
\end{tabular}

this source, the normalization used in Table 2 was with respect to the stress intensity factors for a plane crack of half length $\left(r_{c}+1\right)$. The solution required under 2 seconds overall time for 100 stations and under 10 seconds for 200 stations. A remarkably small difference in the execution times with those recorded for the problem of a buried crack normal to a half-plane surface is probably due to two reasons: efficient algorithms used to evaluate the complete elliptic integrals, and the fact that most of the time is expended on the solution of the linear algebraic system. 
6. Conclusion. The Gauss-Chebyshev quadrature formulae for second-order singular integrals, derived explicitly in this paper, provide a valuable and highly efficient tool for numerical evaluation of finite-part integrals and the solution of hypersingular integral equations. In particular, it has been shown, using examples from fracture mechanics, that the technique provides fast and accurate solutions to the problems of stress intensity factor evaluation, including the cases where the corresponding integral kernel is only known implicitly and requires extremely careful handling when other techniques are used. It is worth noting that numerical implementation of this technique does not require the evaluation of the orthogonal polynomials involved and therefore achieves excellent results using minimal computing effort.

The technique has the potential to be extended to include other weight functions and the corresponding systems of Jacobi polynomials. This may be done using general solutions for interpolatory Gaussian quadratures available in the literature [18]. Specialization of these quadrature rules to specific weight functions and polynomial systems is likely to lead to substantial algorithmic simplifications and savings in computing effort. Relevant applications include those of crack face contact (crack closure), as well as surface-breaking and interface cracks.

7. Acknowledgment. The author would like to express his gratitude to Prof. John Willis and Dr. David Hills for their helpful comments about the manuscript, and to thank a referee for very helpful suggestions.

\section{REFERENCES}

[1] D. L. Clemens and W. T. Ang, Stress intensity factors for the circular annulus crack, Internat. J. Engrg. Sci. 26, 325-329 (1988)

[2] G. Eason, B. Noble, and I. N. Sneddon, On certain integrals of Lipschitz-Hankel type involving products of Bessel functions, Trans. Roy. Soc. 247, 529-551 (1955)

[3] A. Erdelyi, Higher Transcendental Functions, vol. 2, McGraw-Hill, New York, 1953

[4] F. Erdogan, G. D. Gupta, and T. S. Cook, Numerical Solution for Singular Integral Equations, Mechanics of Fracture, vol. 1, Noordhoff, Leiden, 1973, pp. 368-425

[5] I. Gel'fand and V. Shilov, Generalized Functions, Academic Press, 1967

[6] J. Hadamard, Lectures on Cauchy's Problem in Linear Partial Differential Equations, Dover, New York, 1952

[7] D. A. Hills, D. N. Dai, P. A. Kelly, and A. M. Korsunsky, Singular Integral Equations in the Mechanics of Fracture, Kluwer, Dordrecht, 1995

[8] N. I. Ioakimidis and P. S. Theocaris, On the numerical solution of singular integrodifferential equations, Quart. Appl. Math. 37, 325-331 (1979)

[9] A. C. Kaya, Applications of Integral Equations with Strong Singularities in Fracture Mechanics, $\mathrm{Ph}$. D. Thesis, Lehigh University, 1984

[10] A. C. Kaya and F. Erdogan, On the solution of integral equations with strongly singular kernels, Quart. Appl. Math. 45, 105-122 (1987)

[11] A. M. Korsunsky, The Solution of Axisymmetric Crack Problems in Inhomogeneous Media, D. Phil. Thesis, University of Oxford, 1994

[12] A. M. Korsunsky and D. A. Hills, The solution of plane crack problems by dislocation dipole procedures, J. Strain Anal. 30, 21-27 (1995)

[13] A. M. Korsunsky, Fundamental eigenstrain solutions for axisymmetric crack problems, J. Mech. Phys. Solids 43, 1221-1241 (1995)

[14] H. R. Kutt, Quadrature Formulae for Finite-Part Integrals, Spec. Report WISK 178, Nat. Res. Inst. for Math. Sci., Pretoria, 1975 
[15] H. R. Kutt, The numerical evaluation of principal value integrals by finite-part integration, Numer. Math. 24, 205-210 (1975)

[16] M. J. Lighthill, Introduction to Fourier Analysis and Generalized Functions, Cambridge University Press, Cambridge, 1959

[17] G. Monegato, Numerical evaluation of hypersingular integrals, J. Comp. Appl. Math. 50, 9-31 (1994)

[18] G. Monegato, On the weights of certain quadratures for the numerical evaluation of Cauchy principal value integrals and their derivatives, Numer. Math. 50, 273-281 (1987)

[19] Y. Murakami, ed., Stress Intensity Factors Handbook, Pergamon Press, 1987

[20] D. F. Paget, The numerical evaluation of Hadamard finite-part integrals, Numer. Math. 36, 447-453 (1981)

[21] N. J. Salamon and G. G. Walter, Limits of Lipschitz-Hankel integrals, J. Inst. Math. Appl. 24, 237-254 (1979)

[22] G. Szegö, Orthogonal Polynomials, Colloquium Publications, vol. XXIII, Amer. Math. Soc., Providence, RI, 1939 